\title{
Using sparse regularization for multi-resolution tomography of the ionosphere
}

\author{
T. Panicciari, N. D. Smith, C. N. Mitchell, F. Da Dalt, and P. S. J. Spencer \\ Department of Electronic and Electrical Engineering, University of Bath, Bath, BA1 7AY, UK \\ Correspondence to: T. Panicciari (t.panicciari@bath.ac.uk)
}

Received: 4 March 2015 - Published in Nonlin. Processes Geophys. Discuss.: 25 March 2015

Revised: 14 August 2015 - Accepted: 29 August 2015 - Published: 19 October 2015

\begin{abstract}
Computerized ionospheric tomography (CIT) is a technique that allows reconstructing the state of the ionosphere in terms of electron content from a set of slant total electron content (STEC) measurements. It is usually denoted as an inverse problem. In this experiment, the measurements are considered coming from the phase of the GPS signal and, therefore, affected by bias. For this reason the STEC cannot be considered in absolute terms but rather in relative terms. Measurements are collected from receivers not evenly distributed in space and together with limitations such as angle and density of the observations, they are the cause of instability in the operation of inversion. Furthermore, the ionosphere is a dynamic medium whose processes are continuously changing in time and space. This can affect CIT by limiting the accuracy in resolving structures and the processes that describe the ionosphere. Some inversion techniques are based on $\ell_{2}$ minimization algorithms (i.e. Tikhonov regularization) and a standard approach is implemented here using spherical harmonics as a reference to compare the new method. A new approach is proposed for CIT that aims to permit sparsity in the reconstruction coefficients by using wavelet basis functions. It is based on the $\ell_{1}$ minimization technique and wavelet basis functions due to their properties of compact representation. The $\ell_{1}$ minimization is selected because it can optimize the result with an uneven distribution of observations by exploiting the localization property of wavelets. Also illustrated is how the inter-frequency biases on the STEC are calibrated within the operation of inversion, and this is used as a way for evaluating the accuracy of the method. The technique is demonstrated using a simulation, showing the advantage of $\ell_{1}$ minimization to estimate the coefficients over the $\ell_{2}$ minimization. This is in particular
\end{abstract}

true for an uneven observation geometry and especially for multi-resolution CIT.

\section{Introduction}

Tomographic imaging is an important tool for understanding the ionosphere, its behaviour and its effects on radio propagation. Ionospheric disturbances can persist for days in particular conditions. The electron density is the main measure that can tell us about the state of the ionosphere. Enhancements or depletions in the electron density produce irregularities or structures that are present in the ionosphere with different scales and vary with geographical location, time and sun activity. The correct localization of the irregularities can therefore play an important role. The spatial and temporal variability of ionospheric structures justifies the wavelet approach that we will describe in this paper.

CIT (computerized ionospheric tomography) is mainly an underdetermined inverse problem. The goal is to find the spatial and temporal distribution of electron density from a series of relative observations that are collected in the form of slant total electron content (STEC) measured from ground receivers along the signal path. The term relative is introduced because the observations are uncalibrated, hence the calibration becomes part of the inverse problem. Receivers are generally unevenly distributed on the Earth and together with the limited angle geometry of the rays the problem is difficult to solve (Yeh and Raymund, 1991; Na and Lee, 1992) and potentially unstable. Even with increasing observations from new constellations of satellites there is always the problem of the movement of the ionospheric medium in the time taken 
for the Global Navigation Satellite System (GNSS) satellites to cross the sky.

In this type of mathematical problem a functional cost is minimized (Geophysical Inverse Theory and Regularization Problems). A conventional approach is based on Tikhonov regularization (Tikhonov and Arsenin, 1977) and aims to balance the solution for good data agreement and to compensate (regularize) where no data are available. In general a proper regularization is needed to ensure stability, and to reduce artefacts and therefore noise in the reconstruction due to lack of data.

Another recent approach uses the $\ell_{1}$ norm as the metric to regularize the solution. An implementation of this is given by the Fast Iterative Shrinkage-Thresholding Algorithm (FISTA) (Beck and Teboulle, 2009; Daubechies et al., 2004). This algorithm is tailored with wavelets and, under certain conditions, aims to minimize the number of basis functions that can be used to represent the structures in the ionosphere. The efficacy of the algorithm depends on the assumption that the horizontal variation in the ionosphere can be compactly represented with wavelets. It can be a difficult task to prove as we cannot have a real global picture of the ionosphere, but through simulation of the process with a realistic ionospheric model, we can demonstrate that the algorithm works efficiently.

The advantage of having a compact representation is not only in terms of data. It also allows the removal of noise terms (Tsaig and Donoho, 2006), and in the case of ionospheric tomography it can also potentially better handle the uneven data distribution (Schmidt, 2007).

Sparse regularization techniques which minimize the $\ell_{1}$ norm have not been used before in ionospheric tomography and this is what we believe is the first implementation in CIT. The sparse minimization should allow us to exploit more effectively the potential of wavelets to produce a compact reconstruction of the ionosphere. Results from other fields make this technique particularly interesting (see for example, Simons et al., 2011; Loris et al., 2010). Wavelet basis functions have been used in CIT like Haar (Amerian et al., 2010) and B-spline (Durmaz and Karslioğlu, 2011; Schmidt et al., 2008; Zeilhofer et al., 2009) basis functions but they were not used in stabilizing the inversion by means of sparse regularization.

This paper describes an alternative method based on the $\ell_{1}$ norm, using wavelet basis functions, in relation to the $\ell_{2}$ norm, using spherical harmonics, for CIT. The paper will focus on comparing the accuracy of the reconstructions from a simulated ionosphere both quantitatively and qualitatively. The $\ell_{1}$ norm is expected to deal better with the uneven distribution of the observations that we usually encounter in CIT. The properties of wavelets allow the optimizer to select the best combination at different scales and positions according to the data coverage. Small-scale wavelets will be used only where there is good data coverage; this will allow small-scale structures in the ionosphere to be revealed. We used a modi- fied version of the MIDAS (Multi-Instrument Data Analysis System) (Mitchell and Spencer, 2003).

Section 2 gives the definition and the mathematical notations of the problem including biases and basis functions. It also gives an overview of the $\ell_{1}$ and $\ell_{2}$ regularizations that will be used in the paper. Results and conclusions are given in Sects. 4 and 5.

\section{Ionospheric observations}

As in most geophysical applications of tomography, CIT is an undetermined problem. The receiver-satellite geometry and the uneven distribution of the receivers make the inversion a difficult operation. While the vertical sensitivity can be partially improved by means of Empirical Orthonormal Functions (EOFs) (Fremouw et al., 1992; Sutton and Na, 1994), the estimation of horizontal structures can be limited by the presence of artefacts especially when the number of coefficients to estimate increases considerably (e.g. for global or high-resolution maps).

In this section we will firstly define the observations and biases that are involved in the forward problem notation. Then we will describe the inverse problem in terms of basis functions and the regularization techniques.

\subsection{Forward problem}

In CIT observations are collected from ground-based receivers. The measurement $z$ is in the form of Slant Total Electron Content (STEC) defined as the integrated electron content $n$ along the receiver-satellite path

$z=\mathrm{STEC}=\int_{\mathrm{S}_{\mathrm{rX}}}^{\mathrm{s}_{\mathrm{tx}}} n(s) \mathrm{d} s$.

Observations of differential phase can be generally considered noise free from the point of view of the instruments which inherently smooth over noise, but the measurement arc between a single receiver and satellite is uncalibrated or biased. In this section we will discuss the nature of the biases and the mathematical notation we will use to include them in the inversion algorithm.

Equation (1) relates the observation (STEC) with the electron density $n$, which is what is estimated using CIT. In practice, the STEC is obtained by means of dual frequency differences from either the carrier phase of the signal or the pseudoranges of the C/A code (or if available P-code).

Following the Mannucci (Mannucci et al., 1999) notation the recorded pseudorange $P_{i}$ at the frequency $f_{i}(i=1,2)$ can be written as

$P_{i}=\rho+I / f_{i}^{2}+\tau_{i}^{\mathrm{r}}+\tau_{i}^{\mathrm{s}}$,

where the non-dispersive term $\rho$ includes the geometric distance, troposphere delays, clock errors and non-dispersive 
delays in the hardware signal path. The other dispersive delays are frequency-dependent and include the ionospheric delay $I / f_{i}^{2}$ and the dispersive components of the satellite $\tau_{i}^{\mathrm{s}}$ and receiver $\tau_{i}^{\mathrm{r}}$ hardware delays. The ionospheric delay can be separated by differencing Eq. (2) at the two frequencies L1 and L2

$P_{I}=\left(P_{2}-P_{1}\right)=I\left(1 / f_{1}^{2}+1 / f_{2}^{2}\right)+b_{\mathrm{r}}+b_{\mathrm{s}}$,

where $b_{\mathrm{r}}$ and $b_{\mathrm{S}}$ represent the residual frequency-differenced dispersive biases for the receiver and the satellite. Equation (3) has a dependency with the frequency $f_{i}$ of the signal. The relationship between $P_{I}$ and STEC $z$ can be retrieved by substituting a simplified approximation of the AppletonHartree equation (Davies, 1990) and therefore

$I=40.3 z$.

Hence, the STEC can be extracted from Eq. (3) and this can be considered to be in absolute terms where no calibration is required. Unfortunately the multipath-residual biases still contribute as a significant noise component (Jakowski, 1996) which makes the STEC estimations less accurate.

It is possible to use a more accurate estimation of the STEC from the carrier phase but unfortunately with the disadvantage that calibration is required. To explain, and contrasting with the pseudorange in Eq. (2), the carrier phase of the signal $L_{i}$ can be written as

$L_{i}=\rho-I / f_{i}^{2}+\lambda_{i} N_{i}+\varepsilon_{i}^{\mathrm{r}}+\varepsilon_{i}^{\mathrm{s}}$.

The term $N_{i}$ is the integer ambiguity in the phase cycle measurement, and introduces a delay proportional to the wavelength $\lambda_{i}$ of the signal. The ionospheric term $I$ contributes with a negative sign. For this reason it is referred to as phaseadvance. Also in this case, $\varepsilon_{i}^{\mathrm{r}}$ and $\varepsilon_{i}^{\mathrm{s}}$ are the dispersive components of the satellite and receiver hardware delays. As for the code, it is possible to remove the dispersive component $\rho$ by differencing Eq. (5) at the two frequencies L1 and L2

$$
\begin{aligned}
L_{I}=(\mathrm{L} 1-\mathrm{L} 2)= & I\left(1 / f_{1}^{2}+1 / f_{2}^{2}\right)+\left(\lambda_{1} N_{1}-\lambda_{2} N_{2}\right) \\
& +b_{\mathrm{r}}^{\prime}+b_{\mathrm{s}}^{\prime},
\end{aligned}
$$

where $b_{\mathrm{r}}^{\prime}$ and $b_{\mathrm{s}}^{\prime}$ represent the residual frequency-differenced dispersive biases for the receiver and the satellite, and $N_{1}$ and $N_{2}$ are the integer ambiguities in the phase cycle measurements for the frequencies L1 and L2 respectively. The term $\left(\lambda_{1} N_{1}-\lambda_{2} N_{2}\right)$ is generally unknown and introduces a bias that makes the estimated STEC from Eq. (5) a relative measurement. A possible solution to calibrate the GPS-based ionospheric measurements is discussed in different publications (e.g. Mannucci et al., 1999 and Jakowski, 1996) based on a combination of both $P_{I}$ and $L_{I}$. Note that within a short time interval and until the signal loses its lock, the integer ambiguity and the residual terms can be considered constant. This can be used to help calibrate STEC as described before.
However, it is also possible to calibrate the observations directly in the inversion process and this has been shown to have advantages (Dear and Mitchell, 2006), particularly in cases where the hardware biases vary over time. This calibration method will be discussed in the next section.

Measurements for each receiver-satellite pair are contained in the $z$ vector, which is related with the electron content $\boldsymbol{n}$ by the following relationship

$z=\mathbf{A} n+\mathbf{B} b$.

The problem of Eq. (7) is defined on a 3-D grid spacing in altitude, latitude and longitude and it is known as a forwardproblem where $\mathbf{A}$ is the projection matrix that maps the electron content into measurements $z$, and depends on the geometry of the problem. We also included the offset $\boldsymbol{b}$ that takes into account the biases of Eq. (6). The projection matrix $\mathbf{B}$, instead, maps the offset of each ray (observation) into a single offset for each receiver-satellite pair and is defined as

$\mathbf{B}_{i j}= \begin{cases}1 & \text { if } b_{j} \text { is the offset of } z_{i} \\ 0 & \text { otherwise }\end{cases}$

\subsection{Inverse problem}

The quantity we want to estimate is represented in Eq. (7) in terms of electron content $\boldsymbol{n}$ and is obtained through the operation of the inversion. This defines an inverse problem that is generally solved by minimizing the functional $F(\boldsymbol{n}, \boldsymbol{b})$

$$
\begin{aligned}
(\hat{\boldsymbol{n}}, \hat{\boldsymbol{b}}) & =\min (\hat{\boldsymbol{n}}, \hat{\boldsymbol{b}}) F(\boldsymbol{n}, \boldsymbol{b}) \\
F(\boldsymbol{n}, \boldsymbol{b}) & =\|\boldsymbol{z}-\mathbf{A} \boldsymbol{n}-\mathbf{B} \boldsymbol{b}\|^{2}+\alpha \mathcal{P}(\boldsymbol{n}) .
\end{aligned}
$$

The function $\mathcal{P}(\boldsymbol{n})$ defines the regularization (or penalty) term that we need to add to make the inversion a well-posed problem. Equation (10) would not necessarily give a unique solution without $\mathcal{P}(\boldsymbol{n})$ because of the limited-angle geometry and the uneven distribution of the receivers, making the problem ill-conditioned. We are supposing that $\mathcal{P}(\boldsymbol{n})$ operates on the model $\boldsymbol{n}$ only and not on the offsets $\boldsymbol{b}$, which will not be constrained by any assumption coming from the regularization. The parameter $\alpha$ sets a trade-off between the best fitting and the most reasonable stabilization (Zhdanov, 2002). This justifies the different notation of $\hat{\boldsymbol{n}}$ in order to distinguish the approximation from the true $\boldsymbol{n}$.

The functional $F(\boldsymbol{n}, \boldsymbol{b})$ is more computationally expensive and, therefore, is not practically useful. By expanding Eq. (9) with Eq. (10) and after some algebra, Eq. (10) can be rewritten as

$F(\boldsymbol{n})=\|z-\mathbf{A} \boldsymbol{n}\|_{C}^{2}+\alpha \mathcal{P}(\boldsymbol{n})$,

where $\mathbf{C}$ is formed from Laplacian matrices and is defined as

$\mathbf{C}=\mathbf{I}-\mathbf{B}\left(\mathbf{B}^{\mathrm{T}} \mathbf{B}\right)^{-1} \mathbf{B}^{T}$. 
Spencer and Mitchell (2011) described an approach similar to ours; the differences are that in our case an explicit relationship between the estimated biases $\hat{b}$ and the reconstruction $\hat{\boldsymbol{n}}$ is also provided and is given in Eq. (14).

The solution $\hat{\boldsymbol{n}}$ is obtained by minimizing Eq. (14) over $\boldsymbol{n}$, supposing that $\mathcal{P}(\boldsymbol{n})$ is differentiable

$\hat{\boldsymbol{n}}=\left(\mathbf{A}^{\mathrm{T}} \mathbf{C A}+\alpha \frac{1}{2} \frac{\partial \mathcal{P}(\boldsymbol{n})}{\partial \boldsymbol{n}}\right)^{-1} \mathbf{A}^{\mathrm{T}} \mathbf{C} \boldsymbol{z}$.

The solution of Eq. (13) is coincident with the solution we would obtain from Eq. (10). The offsets $\hat{b}$ can then be recovered as

$\hat{\boldsymbol{b}}=\left(\mathbf{B}^{\mathrm{T}} \mathbf{B}\right)^{-1} \mathbf{B}^{\mathrm{T}}(\boldsymbol{z}-\mathbf{A} \hat{\boldsymbol{n}})$.

The observations $z$ have generally a negligible noise term, but in presence of ionospheric structures and because the ionosphere is not a static medium, there could be small variations in STEC even between nearby ray paths. Therefore, the discretization of the ionosphere into a grid is important, which causes the measure $\|\boldsymbol{z}-\mathbf{A} \boldsymbol{n}\|^{2}$ to never reach zero. Therefore a representativity error due to the complexity of the medium is acceptable. What we actually aim for is the minimization of Eq. (11) in order to have the reconstruction matching the observations where data are available up to a residual noisy variation. Therefore the regularization term $\mathcal{P}(\boldsymbol{n})$ becomes the main important term and will be described in Sect. 2.4.

\subsection{Basis functions}

In this section the mathematical notation used to decompose the ionosphere through basis functions is provided. Basis functions are used to extract the information and to emphasize some properties in the reconstructed ionosphere, in this case wave number for spherical harmonics and spatial localization and scale for wavelets. In particular, the vertical profile of electron density is described in terms of basis functions (EOFs) while the horizontal distribution with spherical harmonics and wavelet basis functions. EOFs are obtained from Chapman profiles (Chapman, 1931) and are used to constrain the vertical profile (Hargreaves, 1995). These are taken directly from the standard MIDAS approach as published in 2003.

The inverse problem of Eq. (11) is now expressed, in terms of associated functional, as

$F(\boldsymbol{x})=\|\boldsymbol{z}-\mathbf{A K} \boldsymbol{x}\|_{\mathrm{C}}^{2}+\alpha \mathcal{P}(\boldsymbol{x})$

and solves for the coefficients $x$ of the basis functions, which are contained in the columns of the matrix $\mathbf{K}$. The regularization term $\mathcal{P}(\boldsymbol{x})$ reminds us that the $\boldsymbol{x}$ coefficients are considered regularised instead of the electron density values $\boldsymbol{n}$. The solution becomes the following

$\hat{\boldsymbol{x}}=\left(\mathbf{A}^{\mathrm{T}} \mathbf{K}^{\mathrm{T}} \mathbf{C A K}+\alpha \frac{1}{2} \frac{\partial \mathcal{P}(\boldsymbol{x})}{\partial \boldsymbol{x}}\right)^{-1} \mathbf{K}^{\mathrm{T}} \mathbf{A}^{\mathrm{T}} \mathbf{C} z$

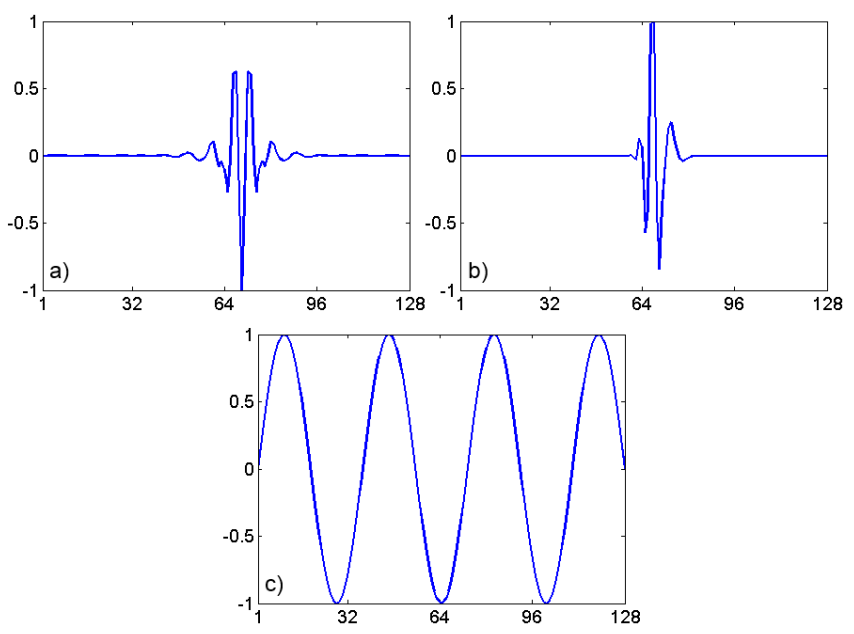

Figure 1. (a) Discretized Meyer basis function for a particular scale and translation; (b) Daubechies (4 tap) basis function with the same scale and translation as (a); (c) a Fourier sinusoid component of the spherical harmonic basis functions. Basis functions are shown normalized to one and are interpolated for ease of viewing.

and the offsets $\hat{\boldsymbol{b}}$ can then be recovered as

$\hat{\boldsymbol{b}}=\left(\mathbf{B}^{\mathrm{T}} \mathbf{B}\right)^{-1} \mathbf{B}^{\mathrm{T}}(\boldsymbol{z}-\mathbf{A} \mathbf{K} \hat{\boldsymbol{x}})$.

The choice of $\mathbf{K}$ has been limited to orthogonal basis functions for this paper.

Figure 1 shows a one-dimensional example of the basis functions (normalized to one) that will be used in the experiment. Figure $1 \mathrm{a}$ and $\mathrm{b}$ illustrate two wavelets at the same scale and position for discrete Meyer (DM) and Daubechies 4 (DB4). They have a spatial compact support that makes them particularly useful to resolve localized structures. Figure 1c shows a single harmonic (normalized to one) that has to be multiplied with the Lagrange polynomial (along latitude) to produce a spherical harmonic (SH). They have a longer spatial support and work well to describe periodicities in the ionosphere.

\subsection{Regularization}

Different regularizations exist to stabilize Eq. (15) and make the solution unique and physically meaningful. In this section the two regularizations based on the $\ell_{1}$ and $\ell_{2}$ norm, which are both used for the reconstructions in Sec. 3.1, will be described.

The main goal of regularization is finding the best representation of the ionosphere that matches the observations and at the same time obviates the lack of data we usually face (e.g. in the oceans between continents).

Regularization techniques exploit the fact that Eq. (15) can be convex, i.e. that by minimizing it a global minimum is guaranteed, but it does not mean that different regularizations may have the same minima. The minimizer becomes the best 
representation we can have, and its properties will strongly depend on the chosen regularization term.

The $\ell_{1}$ and $\ell_{2}$ regularizations used in this work both aim to create a sufficiently detailed solution by maintaining as much information as possible from the observations. The difference lies in the information that can be extracted from the observations through basis functions and, therefore, on the efficiency on resolving different scale structures. For example, wavelets are good to localize structures, while spherical harmonics works well with periodicities. Therefore, we are expecting wavelets to resolve better localized structures than spherical harmonics.

The regularization term of Eq. (15) can be expressed in different ways. The classical approach is by using an $\ell_{2}$ norm (or Tikhonov regularization)

$\mathcal{P}(\boldsymbol{x})=\|\mathbf{P K} \boldsymbol{x}\|_{2}^{2}$,

where the matrix $\mathbf{P}$ is used to select only a subspace of the possible solutions and stabilize the problem of Eq. (15) toward a physically acceptable solution, and the $\ell_{2}$ norm is defined as $\|\boldsymbol{a}\|_{2}=\sqrt[2]{\sum_{i}\left|a_{i}\right|^{2}}$. In the implementation of this paper $\mathbf{P}$ is set to the identity matrix and the minimization of Eq. (15) with Eq. (18) is solved with the LU decomposition similarly to the framework in Mitchell and Spencer (2003). Another suitable choice of $\mathbf{P}$ can be the Laplacian matrix.

A different measure comes from the sparsity which involves the number of nonzero coefficients in $\boldsymbol{x}$ (Bruckstein et al., 2009) and is obtained with

$\mathcal{P}(\boldsymbol{x})=\|\mathbf{K} \boldsymbol{x}\|_{0}$,

where $\|\boldsymbol{a}\|_{0}$ stands for the number of coefficients $a_{i}$ that are not zero. This approach is particularly suitable for wavelets as it exploits their ability for compact representation. By minimizing Eq. (15) with Eq. (19) we are looking for the solution which produces the best agreement with the observations but that at the same time is also the sparsest one. The minimization with Eq. (19) is unfortunately not convex, which means that it may have local minima. The complexity of this problem was also proven to be in general not practical as the solution requires to exhaustively search for all the possible combinations of basis functions (the columns in $\mathbf{K}$ ) that minimize the functional of Eq. (15) (Natarajan, 1995).

A more feasible solution is obtained with the $\ell_{1}$ norm, which in some sense is half way between Eq. (18) and Eq. (19)

$\mathcal{P}(\boldsymbol{x})=\|\mathbf{P K} \boldsymbol{x}\|_{1}$,

where $\|\boldsymbol{a}\|_{1}=\sum_{i}\left|a_{i}\right|$. The regularization term of Eq. (20) makes the functional Eq. (15) convex and therefore makes it possible to have a unique solution. The solution may differ from the one obtained by Eq. (19), but there are mathematical conditions that ensure Eq. (20) to be equivalent to Eq. (19) producing an identical solution up to an error term that is proportional to the input noise level (Donoho et al., 2006; Donoho, 2006). The whole theory requires that $\boldsymbol{x}$ is sufficiently sparse, i.e. that the ionosphere can be represented with few basis functions. According to this, sparsity becomes the main goal, allowing better noise removal (i.e. false artefacts in the reconstruction) but also better compression (e.g. for storing data) in respect to the non-sparse solution (Tsaig and Donoho, 2006; Schmidt, 2007).

The minimization of Eq. (15) with Eq. (20) is implemented with the Fast Iterative Shrinkage-Thresholding Algorithm (FISTA, see Beck and Teboulle, 2009). It applies a non-linear thresholding (or soft-thresholding) (Donoho and Johnstone, 1994) to the estimated coefficients $\hat{\boldsymbol{x}}_{i}$ at the $i$ th iteration

$\eta\left(\hat{\boldsymbol{x}}_{i}\right)=\left\{\begin{array}{ll}0 & \text { if }\left|\hat{\boldsymbol{x}}_{i}\right| \leq \alpha \xi \\ \operatorname{sgn}\left(\hat{\boldsymbol{x}}_{i}\right)\left(\left|\hat{\boldsymbol{x}}_{i}\right|-\alpha \xi\right) & \text { if }\left|\hat{\boldsymbol{x}}_{i}\right|>\alpha \xi\end{array}\right.$,

where $\xi$ is set to the reciprocal of the maximum eigenvalue of $\mathbf{K}^{\mathrm{T}} \mathbf{A}^{\mathrm{T}} \mathbf{A} \mathbf{K}$ and together with $\alpha$ sets the threshold. Then the estimation at the next iteration is $\hat{\boldsymbol{x}}_{i+1}=\eta\left(\hat{\boldsymbol{x}}_{i}\right)$.

A similar stabilization can be introduced by selecting a subset of $\mathbf{K}$ by means of a similar thresholding or a greedy subset selection directly applied to Eqs. (15) and (18). This was shown to be not optimal in a general case (Donoho, 2006).

\section{Simulation}

We selected a grid that spans from North America to Europe. This is a good example to show the limitation imposed, in this case by the ocean, on the density of the receivers. We selected a grid of dimension $64 \times 64$ voxels in longitude and latitude, and 22 voxels in altitude. It produces a voxel of dimension around $1 \times 2^{\circ}$ in latitude and longitude and $50 \mathrm{~km}$ in altitude.

Data were simulated with the international reference ionosphere (IRI) model. Some structures were then added in order to test the efficiency of the algorithm to resolve them. We considered uncalibrated observations that were obtained by adding a constant bias to each receiver-satellite pair and we collected observations within a time window of 8 min with a sample rate of $30 \mathrm{~s}$. Although the inversion method is not time-dependent, there is the necessity to collect data within a relatively short time window (in our case $8 \mathrm{~min}$ long). The reason for the time window was to increase the data coverage otherwise there would be insufficient data for a reliable inversion. Effectively this assumes the ionosphere is static over $8 \mathrm{~min}$, which can be considered more valid during quiet ionospheric conditions. However, with the anticipated increase in GNSS satellite numbers the size of the time window could be reduced. Representativity error was taken into account by adding a term to the observations distributed as a Gaussian noise. This term also takes into account any non-dispersive residual term described in Eq. (6). 


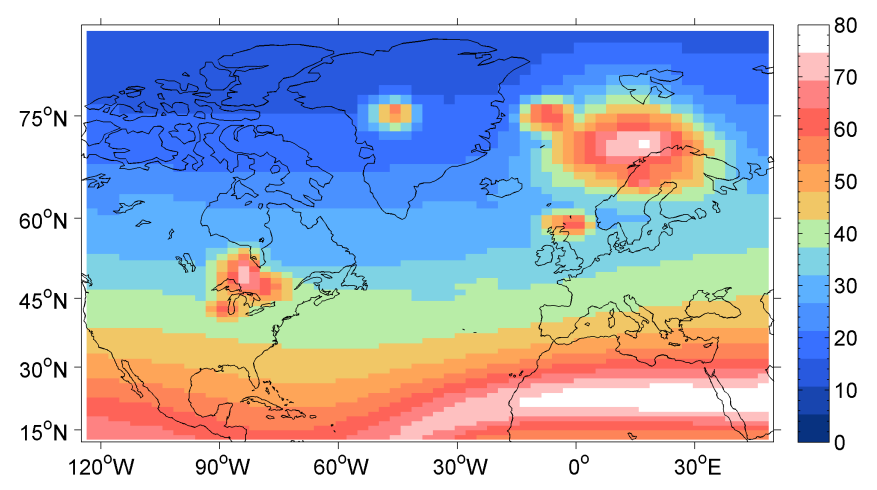

Figure 2. Simulated ionosphere with structures added to IRI2012. Values are in TECU $\left(10^{16}\right.$ electronsperm $\left.^{-2}\right)$.

Figure 2 shows the Vertical Total Electron Content (VTEC) map that was used as truth while Fig. 3 illustrates the number of rays that was used in the reconstruction (black dots are the ground stations). The number of rays is obtained by summing the intersections along the altitude within voxels of the grid. The VTEC is calculated by integrating the electron content in a certain latitude and longitude location along the altitude. The ray coverage strictly depends on the density of ground stations, data (STEC) sampling rate and, in our case, the time window within which we run the reconstruction. The selection of the grid is also important as a finer grid will increase the number of voxels that are not intercepted by a ray and the number of coefficients to estimate.

Some structures were located where data coverage is particularly low. In those locations the reconstruction will struggle to recover the actual value independently from the regularization that has been used. The behaviour of the algorithm in those zones will strongly depend on the regularization term.

We used EOFs obtained from Chapman profiles (Hargreaves, 1995), and wavelets (DB4 and DM) and Spherical Harmonics $(\mathrm{SH})$ to represent the horizontal distribution of structures in the ionosphere. By selecting a subset of larger horizontal basis functions we also limited the resolution in the reconstruction, i.e. the smallest scale structures that can be resolved.

For the aim of this paper it will be considered a standard implementation of Eqs. (18) and (20), i.e. the matrix $\mathbf{P}$ will be set to the identity matrix.

\subsection{Inversion}

The reconstructions are shown in Fig. 4 for low resolution and Fig. 5 for high resolution. Each figure shows the behaviour of the algorithm using different basis functions: $\mathrm{SH}$ (top), DM (middle) and DB4 (bottom). In order to highlight the regularization effects where only data coverage was present, we applied a mask (left) to the reconstruction (right). In fact, each regularization technique will handle the absence

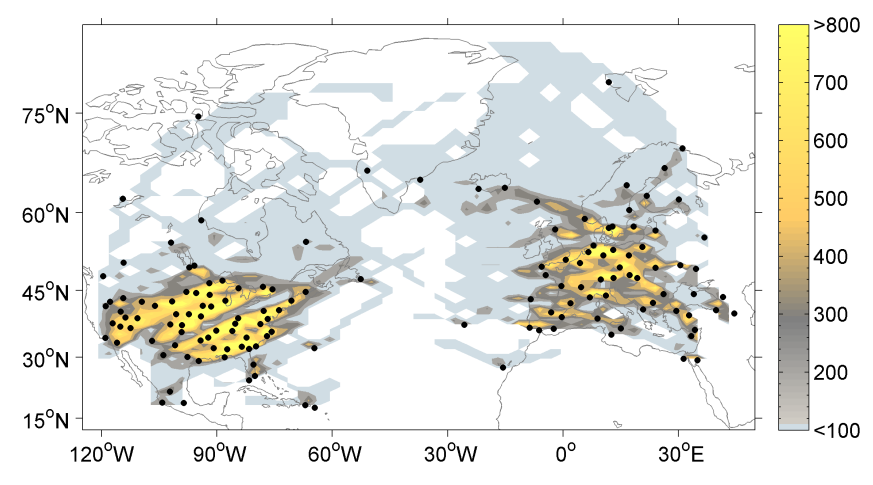

Figure 3. Number of rays with ground stations (black dots).

of data in different ways but we want to compare their ability to resolve structures where data are available.

At low resolution the reconstruction looks reasonable for both methods. The structures appear smoothed and with little detail (Fig. 4a, b, c). SH seems to produce some oscillations outside the data coverage (Fig. 4d), mainly in the Atlantic Ocean. This is due to the sinusoidal nature of SH that makes it problematic to represent localized structures. Wavelets do not produce oscillations and the reconstruction looks reasonably smoothed for this resolution, but there are some edge effects, especially for DM, between Canada and Greenland. Furthermore, DB4 unlike DM tends to fill the data gap in the ocean (Fig. 4f).

As the resolution increases (and therefore the number of coefficients to estimate) the inversion needs in general a stronger regularization. This is shown in Fig. 5. With SH the regularization damps many coefficients down but it seems to resolve some of the structures well (north UK and US) where good data coverage is present (Fig. 5a, b, c). However the reconstruction presents the ring oscillation phenomenon that is an indication of the limitation of the method when a high number of basis functions are used (Fig. 5d). The stronger regularization has reduced most of the coefficients, and the VTEC is in general underestimated. In fact, we are expecting a VTEC of 40 in central Europe but the reconstruction shows a VTEC less than 30. Where data are not available the regularization forces the VTEC to go rapidly toward zero. With wavelets the regularization aims to minimize the number of non-zero coefficients. Therefore the smallest basis functions are contributing with the largest (smoother) ones to add detail to the reconstruction only where good data coverage is available (this concept is regarded as multi-resolution, which will be explained later). Where data are not enough to resolve a small structure the solution will be approximated with a bigger and smoothed one. By looking at the VTEC values, wavelets are perfectly recovering the value of 40 VTEC units in Europe (Fig. 5b, c). This is mainly due to the fact that the regularization term, by exploiting the localization properties of wavelets, is adding the smallest basis only if they detect a significant enhancement over the threshold $\alpha \xi$ of Eq. (21). In 

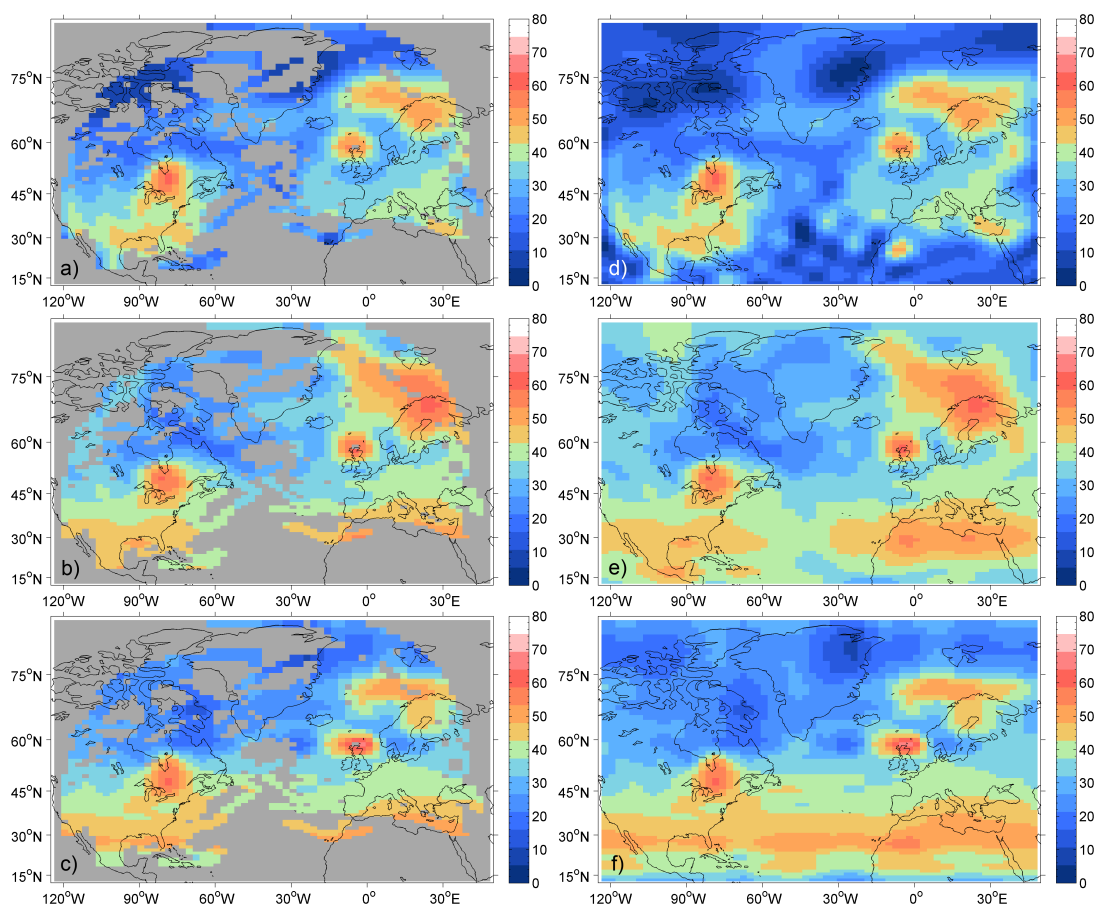

Figure 4. Reconstructions obtained at low resolution with masked out VTEC values where there is no ray coverage for (a) spherical harmonics; (b) discrete Meyer; (c) Daubechies 4; and without the mask for (d) spherical harmonics; (e) discrete Meyer; (f) Daubechies 4. Values are in TECU $\left(10^{16}\right.$ electronsperm $\left.^{-2}\right)$.
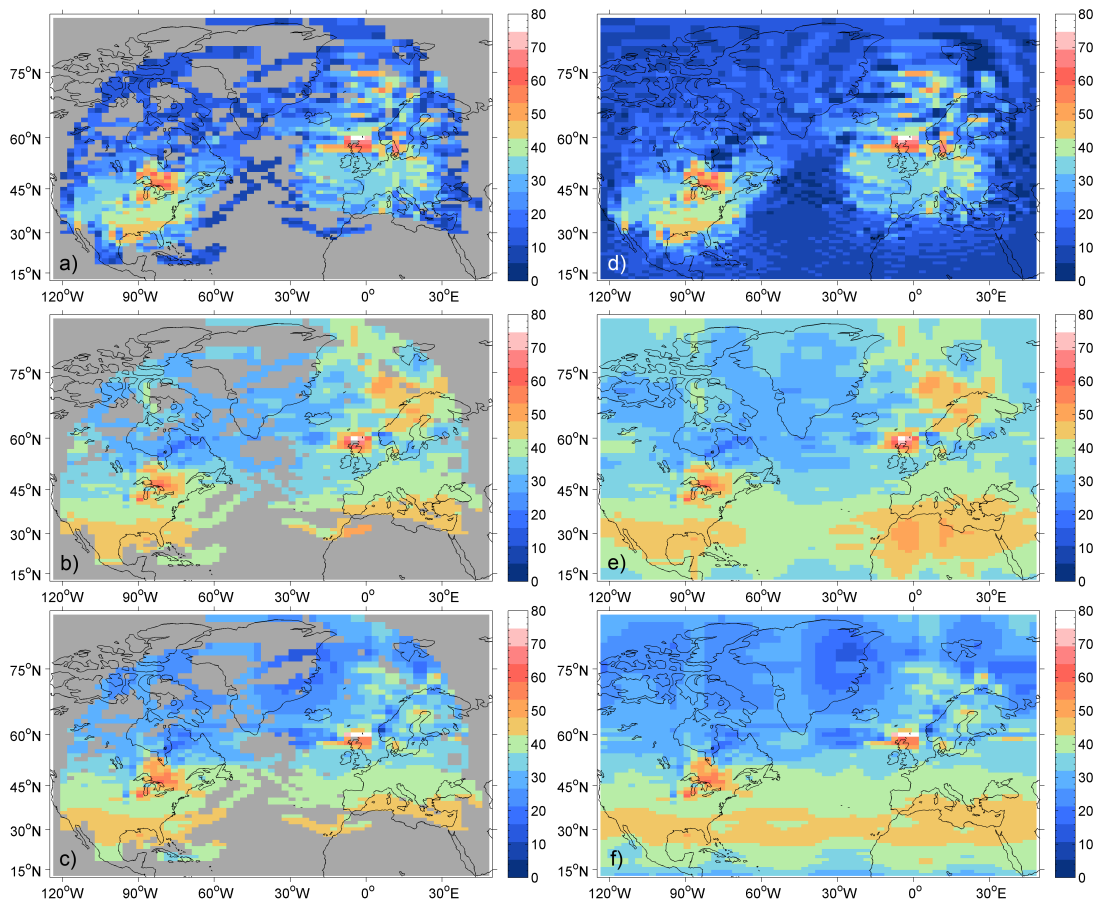

Figure 5. Reconstructions obtained at high resolution with masked out VTEC values where there is no ray coverage for (a) spherical harmonics; (b) discrete Meyer; (c) Daubechies 4; and without the mask for (d) spherical harmonics; (e) discrete Meyer; (f) Daubechies 4. Values are in TECU $\left(10^{16}\right.$ electronsperm $\left.^{-2}\right)$.

general the VTEC variation is well recovered with wavelets

and they seem to produce the best estimation of the ionosphere. 
Table 1. RMS error (values are in TECU) of the VTEC map obtained with spherical harmonics and wavelets at two different resolutions. Only the VTEC coefficients where there is ray coverage were considered. The percentage of basis functions with non-zero coefficients is also shown and, within brackets, the number in absolute value.

\begin{tabular}{lcccccc}
\hline & \multicolumn{2}{c}{ Low resolution } & & \multicolumn{2}{c}{ High resolution } \\
\cline { 2 - 3 } \cline { 5 - 6 } & $\begin{array}{c}\text { RMS error } \\
(\text { TECU) }\end{array}$ & $\begin{array}{c}\text { Number of basis } \\
\text { functions }\end{array}$ & & $\begin{array}{c}\text { RMS error } \\
(\text { TECU) }\end{array}$ & $\begin{array}{c}\text { Number of basis } \\
\text { functions }\end{array}$ \\
\hline Spherical harmonics & 10.87 & $100 \%(1089)$ & & 19.32 & $100 \%(16641)$ \\
Discrete Meyer & 6.66 & $36 \%(92)$ & & 8.5 & $8 \%(317)$ \\
Daubechies 4 & 7.48 & $41 \%(106)$ & & 8.59 & $11 \%(467)$ \\
\hline
\end{tabular}

For each reconstruction the root mean square (RMS) error of the VTEC between the true and the reconstructed ionosphere was calculated. The RMS error is taking into account only the VTEC values where there is ray coverage. Values where there is no ray are, in fact, less meaningful for this statistic.

Table 1 shows the RMS error and the number of basis functions for each reconstruction at the two different resolutions. The number of basis functions is shown in percentage and in absolute values within the brackets. The increasing of RMS error with resolution is caused by the attempt of the basis functions to describe the small variations in STEC due to non-uniform data coverage (especially in north Norway). Wavelets need less than $50 \%$ of basis functions at low resolution and even less at high resolution. The small number of basis functions help to stabilize the inversion as only fewer coefficients have to be estimated.

The offsets, obtained from Eq. (14) and averaged for each receiver, are also very well recovered at low resolution by $\mathrm{SH}$ (Fig. 6a) and DM (Fig. 6b). Figure 7a and b show the scatter plot of the original offsets ( $x$ axis) and the estimated ones ( $y$ axis) for each receiver, obtained from the high resolution reconstruction using $\mathrm{SH}$ and DM basis functions. At high resolution the offsets are still well estimated from wavelets (Fig. 7b) while they seem to be biased with SH (Fig. 7a). There is in general an overestimation of the offsets that increases as the regularization coefficient $\alpha$ increases. This is due to the fact that when $\alpha$ increases, the difference between the observations and the estimation in Eq. (14) increases as well as making the offsets bigger.

\subsection{Multi-resolution map}

As introduced earlier, another concept that can be exploited with wavelets is multi-resolution analysis. A similar concept was already used in (Schmidt, 2007). Wavelets allow the detection of structures according to their scale and position. Small-scale basis functions are therefore selected to represent small variations, otherwise only the basis functions with bigger scales are used. The ability of the algorithm to recognize small variations depends on the data availability and, therefore, the resolution (here intended as the smallest scale

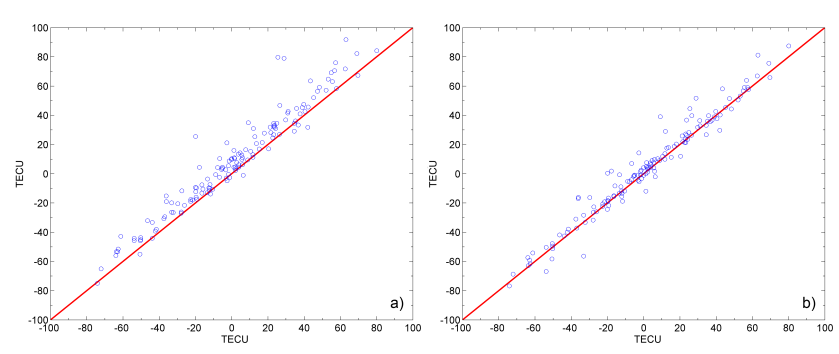

Figure 6. Scatter plot of the estimated offsets ( $y$ axis) versus the true offsets ( $x$ axis) with (a) spherical harmonics and (b) discrete Meyer at low resolution.
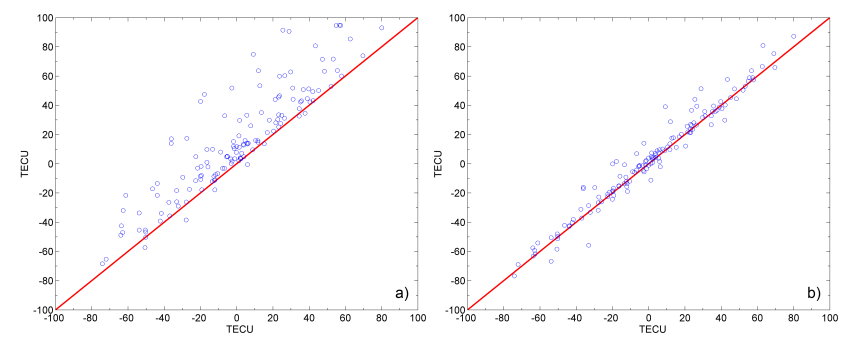

Figure 7. Scatter plot of the estimated offsets ( $y$ axis) versus the true offsets ( $x$ axis) with (a) spherical harmonics and (b) discrete Meyer at high resolution.

we can resolve in a certain position in the map) will depend on data.

Figure 8 aims to explain multi-resolution with DM basis functions. Each square box indicates where the wavelet is centered in the map and the size that the wavelet is contributing with (i.e. the scale of the wavelet, which we selected the same level for each box). This is valid only in principle as a wavelet can be defined in a longer domain than the one defined by the square. The algorithm selects smaller scale basis functions where data coverage is good, trying, as a consequence, to match better the observations. In regions where data are not available or not enough, only the biggest scale wavelets are selected and therefore the solution will look smoother. This is not possible to obtain with $\mathrm{SH}$ as they are longer functions and are defined over the whole globe. It is interesting to notice how small-scale wavelets are not used 


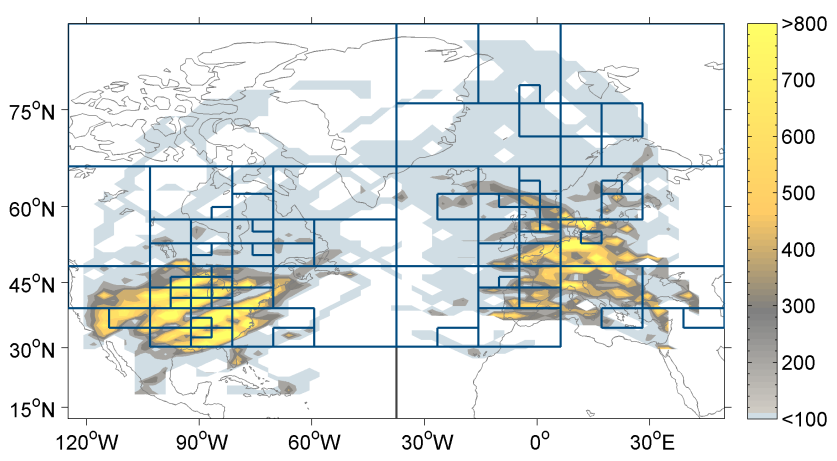

Figure 8. Multi-Resolution (MR) Map for the high-resolution case with discrete Meyer basis functions. Each box represents the scale of the basis function and its position.

if there is not a comparable (to the scale of the wavelet) enhancement from the data. This is the case in east and south Europe where, even if good data coverage is provided, only large-scale wavelets are used.

\subsection{Noise sensitivity}

We stated at the beginning that wavelets allow the better removal of noisy terms in the reconstruction. Actually ground stations produce observations that can be considered generally noiseless. The noise term that we intended comes from the fact that the ionosphere is a dynamic medium, where different scale structures evolve with time according to complicated physics laws in a complex environment.

In order to test the effect of variability in the observations, we decided to add a zero mean Gaussian noise to each observation with a standard deviation of 1 TEC unit. A similar approach was used by Chartier et al. $(2012,2014)$.

Figure 9a and $\mathrm{b}$ show the reconstruction obtained with $\mathrm{SH}$ and DM. SH reconstruction (Fig. 9a) is quite sensitive to the noise, which causes additional oscillations and artefacts. DM reconstruction (Fig. 9b) shows a better robustness to noise, and the reconstruction is similar to the ones in Fig. 5e-f. This is mainly due to the sparse regularization which aims to minimize the number of nonzero coefficients. When the soft-thresholding of Eq. (20) is applied with FISTA, a subset of the most significant coefficients is selected. Those coefficients will contain the most important part of the energy (or information) (Donoho and Johnstone, 1994). In general, it would not be possible to make the same considerations if the energy was evenly distributed among all the coefficients, like in the case of SH.

The RMS error obtained from Fig. 9a and $\mathrm{b}$ is shown also in this case in Table 2 together with the percentage of number of basis functions with non-zero coefficients. The number of basis functions used with DM is slightly decreased compared to the case without noise. This is due to the higher threshold $\alpha \xi$ (see Eq. 21) that we used to remove the noise. In the case
Table 2. RMS error (values are in TECU) of the VTEC map obtained with spherical harmonics and discrete Meyer with a noise term added to the observations. Only the VTEC coefficients where there is ray coverage were considered. The percentage of basis functions with non-zero coefficients is also shown and, within brackets, the number in absolute value.

\begin{tabular}{lcc}
\hline & $\begin{array}{c}\text { RMS } \\
\text { error } \\
\text { (TECU) }\end{array}$ & $\begin{array}{c}\text { Number } \\
\text { of basis } \\
\text { functions }\end{array}$ \\
\hline Spherical harmonics & 24.45 & $100 \%(16641)$ \\
Discrete Meyer & 9.35 & $7 \%(282)$ \\
\hline
\end{tabular}

of SH all the basis functions are still used although a higher regularization parameter $\alpha$ was used too.

\subsection{Model-aided inversion}

We implemented a model-aided inversion by imaging the residual after removing from the observations a background model of the ionosphere. This is called three-dimensional variational (3DVar) data assimilation and assumes the knowledge of a priori information about the state of the ionosphere. This is generally obtained with an empirical model (like IRI2012) or a first principle physics model. For the sake of this paper we wanted to test the algorithms with Eqs. (18) and (20) under these conditions. Therefore, we considered there was almost perfect knowledge of the ionosphere, i.e. we set the background model $\boldsymbol{n}_{0}$ to IRI2012 (without the added structures) and considered the residual $\delta \boldsymbol{n}$

$\delta \boldsymbol{n}=\boldsymbol{n}-\boldsymbol{n}_{0}$.

This residual is associated with a residual $\delta z$ in the measurements $z$ calculated as

$\delta z=z-\mathbf{A} \boldsymbol{n}_{0}$.

Therefore, the problem in Eq. (15) becomes

$F(\delta \boldsymbol{x})=\|\delta \boldsymbol{z}-\mathbf{A} \mathbf{K} \delta \boldsymbol{x}\|_{C}^{2}+\alpha \mathcal{P}(\delta \boldsymbol{x})$,

where $\delta \boldsymbol{x}=\left(\mathbf{K}^{\mathrm{T}} \mathbf{K}\right)^{-1} \mathbf{K}^{\mathrm{T}} \delta \boldsymbol{n}$. Hence, the inverse problem is applied to Eq. (24), which will calculate the residual information that the a priori model could not reproduce (in this case the structures added to IRI2012). The final reconstruction is obtained by summing the estimated $\delta \hat{\boldsymbol{n}}$ to the background model $\boldsymbol{n}_{0}$. To make the problem more difficult we also added the noise term into the data $z$ as in the previous section. The reconstruction (plus background model) is shown in Fig. 10a and $\mathrm{b}$ for $\mathrm{SH}$ and DM.

As we expected both methods work well. The only remarkable difference is that $\mathrm{SH}$ basis functions are picking up some noisy coefficients which result in a noisier reconstruction than with DM. Table 3 summarizes the RMS error obtained for these reconstructions. 

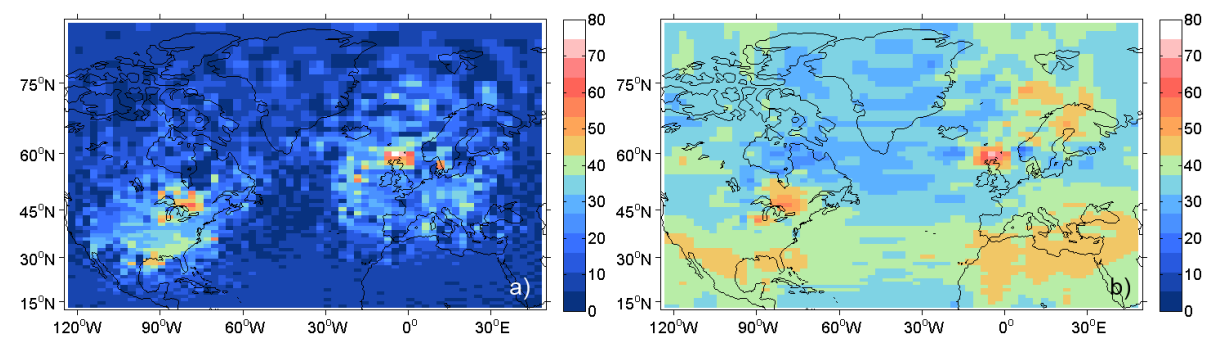

Figure 9. Reconstruction with (a) spherical harmonics and (b) discrete Meyer at high resolution with Gaussian noise added to the observations. The Gaussian noise has zero mean and a standard deviation of 1 TEC unit.
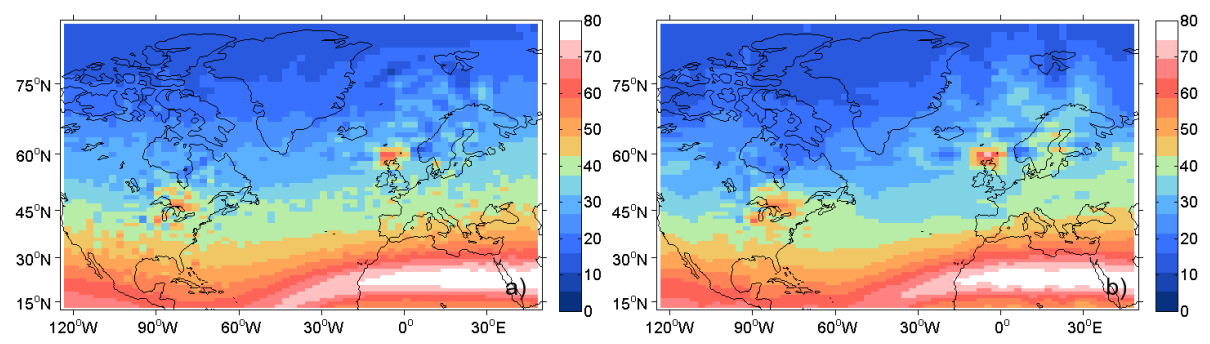

Figure 10. Model-aided reconstruction obtained with (a) spherical harmonics and (b) discrete Meyer at high resolution. A noise term (zeromean Gaussian with 1 TEC unit of standard deviation) was added to the observations.

Table 3. RMS error (values are in TECU) of the VTEC map obtained with a 3DVar scheme using spherical harmonics and discrete Meyer with a noise term added to the observations. Only the VTEC coefficients where there is ray coverage were considered. The percentage of basis functions with non-zero coefficients is also shown and within brackets the number in absolute value.

\begin{tabular}{lrr}
\hline & $\begin{array}{r}\text { RMS } \\
\text { error } \\
\text { (TECU) }\end{array}$ & $\begin{array}{r}\text { Number } \\
\text { of basis } \\
\text { functions }\end{array}$ \\
\hline Spherical harmonics & 9.26 & $100 \%(16641)$ \\
Discrete Meyer & 8.66 & $7.3 \%(300)$ \\
\hline
\end{tabular}

By perfectly removing the background the algorithm needs to resolve only few relatively smooth structures at a different scale. This scenario can be considered as the best case, where we had background knowledge of the ionosphere, in comparison with the worst case of the previous subsection where such knowledge was lacking. Actually, we will never have a perfect knowledge of the ionosphere and, therefore, a background model cannot aid the reconstruction as in the above example. This mismatching with the truth means that the algorithm with an approximated background model will have performances between the worst and best case.

\section{Conclusions}

Sparse regularization has been shown to be a valid alternative to standard method based on Tikhonov regularization and is particularly suitable with wavelets.

The method has been tested to estimate the offsets of the observations and, even though it was applied to the specific case of the computerized ionospheric tomography (CIT), it can be used for general inverse problems where unknown offsets must be estimated. The method gave good performances in recovering the offsets, but a useful remark is that there is a tendency to overestimate them as $\alpha$ increases. When $\alpha$ increases the difference between the estimation and the observations increases, making the offsets bigger (Eq. 17). This is probably due to the absence of regularization into the estimated offsets (Eq. 11). Previous works (Dear and Mitchell, 2006; Chartier et al., 2012) used a calibration matrix similar to the one defined in Eq. (12) to estimate the offsets from regional ionospheric reconstructions. They compared estimated offsets from tomographic reconstructions using an independent data set from the Center for Orbit Determination in Europe (CODE). Both the previous works showed a good match of the estimated offsets with CODE and did not exhibit any bias. The main objective here is, instead, to reproduce high-resolution maps from global ionospheric reconstructions. This involves the estimation of a higher number of coefficients and dealing with more uneven data distribution and data gaps (e.g. oceans). Under those conditions the choice of the regularization becomes of primary importance.

Sparsity allows a better noise removal and a more stable regularization when the number of coefficients to estimate 
increases considerably. We have shown tomographic reconstructions obtained with Spherical Harmonics ( $\mathrm{SH}$ ) and two different wavelets, Daubechies 4 (DB4) and Discrete Meyer (DM) in a worst and best case. The best case was obtained by selecting a background model which exactly represented the smoothed ionosphere, whilst the worst case was without any background model. In both cases wavelets were shown to produce the best reconstruction in terms of the root mean square (RMS) error and oscillations (artefacts). An important characteristic in this new approach is the ability of wavelets to handle the uneven distribution of the observations. We have explained this ability through the multi-resolution map showing how the resolution is adapted to the data coverage and the ionospheric structures observed by the measurements.

It is noted that CIT is actually a time-dependent inversion problem and in this paper it has been simplified in the simulation to a case where the ionosphere does not change in time. The work in this paper has shown the potential of the method when the ionosphere does not considerably change within a short time window, e.g. under quiet geomagnetic conditions. For more active conditions a full 4-D imaging would be required. This factor will be studied in further research.

In conclusion sparse regularization techniques can produce significant improvements to CIT and to inverse problems in general. They demonstrate properties of noise robustness and adaptability to data coverage. The choice of wavelet basis functions is not critical, but we believe that other wavelet constructions could lead to further improvements.

Acknowledgements. We thank the International Reference Ionosphere (IRI) project (http://iri.gsfc.nasa.gov/) and the UNAVCO and IGS repositories for offering free access to GPS data. This research activity was funded by the European Union's Seventh Framework Programme for research, technological development and demonstration under grant agreement no. 264476.

Edited by: G. Lapenta

Reviewed by: two anonymous referees

\section{References}

Amerian, Y., Hossainali, M. H., Voosoghi, B., and Ghaffari, M. R.: Tomographic Reconstruction of the Ionospheric Electron Density in term of Wavelets, Journal of Aerospace Science and Technology, 7, 19-29, 2010.

Beck, A. and Teboulle, M.: A fast iterative shrinkage-thresholding algorithm for linear inverse problems, SIAM Journal on Imaging Sciences, 2, 183-202, 2009.

Bruckstein, A. M., Donoho, D. L., and Elad, M.: From Sparse Solutions of Systems of Equations to Sparse Modeling of Signals and Images, SIAM Review, 51, 34-81, doi:10.1137/060657704, 2009.
Chapman, S.: The absorption and dissociative or ionizing effect of monochromatic radiation in an atmosphere on a rotating earth, P. Phys. Soc., 43, 26-45, 1931.

Chartier, A. T., Mitchell, C. N., and Jackson, D. R.: A 12 year comparison of MIDAS and IRI 2007 ionospheric Total Electron Content, Adv. Space Res., 49, 1348-1355, doi:10.1016/j.asr.2012.02.014, 2012.

Chartier, A. T., Kinrade, J., Mitchell, C. N., Rose, J. A. R., Jackson, D. R., Cilliers, P., Habarulema, J.-B., Katamzi, Z., McKinnell, L.-A., Matamba, T., Opperman, B., Ssessanga, N., Giday, N. M., Tyalimpi, V., Franceschi, G. D., Romano, V., Scotto, C., Notarpietro, R., Dovis, F., Avenant, E., Wonnacott, R., Oyeyemi, E., Mahrous, A., Tsidu, G. M., Lekamisy, H., Olwendo, J. O., Sibanda, P., Gogie, T. K., Rabiu, B., Jong, K. D., and Adewale, A.: Ionospheric imaging in Africa, Radio Sci., 49, 19-27, doi:10.1002/2013RS005238, 2014

Daubechies, I., Defrise, M., and De Mol, C.: An iterative thresholding algorithm for linear inverse problems with a sparsity constraint, Commun. Pur. Appl. Math., 57, 1413-1457, doi:10.1002/cpa.20042, 2004

Davies, K.: Ionospheric Radio, in: Ieee Electromagnetic Waves Series (Book 31), The Institution of Engineering and Technology, London, 1990

Dear, R. M. and Mitchell, C. N.: GPS interfrequency biases and total electron content errors in ionospheric imaging over Europe, Radio Sci., 41, RS6007, doi:10.1029/2005RS003269, 2006.

Donoho, D. L.: For most large underdetermined systems of linear equations the minimal $\ell^{1}$-norm solution is also the sparsest solution, Commun. Pur. Appl. Math., 59, 797-829, doi:10.1002/cpa.20132, 2006.

Donoho, D. L. and Johnstone, I. M.: Threshold selection for wavelet shrinkage of noisy data, in: Engineering in Medicine and Biology Society, 1994. Engineering Advances: New Opportunities for Biomedical Engineers. Proceedings of the 16th Annual International Conference of the IEEE, 3-6 November 1994, Baltimore, MD, A24-A25, 1994.

Donoho, D. L., Elad, M., and Temlyakov, V. N.: Stable recovery of sparse overcomplete representations in the presence of noise, IEEE T. Inform. Theory, 52, 6-18, doi:10.1109/TIT.2005.860430, 2006.

Durmaz, M. and Karslioğlu, M. O.: Non-parametric regional VTEC modeling with Multivariate Adaptive Regression B-Splines, Adv. Space Res., 48, 1523-1530, doi:10.1016/j.asr.2011.06.031, 2011.

Fremouw, E. J., Secan, J. A., and Howe, B. M.: Application of stochastic inverse theory to ionospheric tomography, Radio Sci., 27, 721-732, doi:10.1029/92RS00515, 1992.

Hargreaves, J. K.: The Solar-Terrestrial Environment: An Introduction to Geospace - The Science of the Terrestrial Upper Atmosphere, Ionosphere, and Magnetosphere, in: Cambridge Atmospheric and Space Science Series, Cambridge University Press, New York, 1995.

Jakowski, N.: TEC monitoring by using satellite positioning systems, in: Modern Ionospheric Science - 50 Years of Ionospheric Research in Lindau, edited by: Kohl, H., Rüster, R., and Schlegel, K., Produserv GmbH Verlagsservice, Germany, 371-389, 1996.

Loris, I., Douma, H., Nolet, G., Daubechies, I., and Regone, C.: Nonlinear regularization techniques for seismic tomography, 
J. Comput. Phys., 229, 890-905, doi:10.1016/j.jcp.2009.10.020, 2010.

Mannucci, A. J., Iijima, B. A., Lindqwister, U. J., Pi, X., Sparks, L., and Wilson, B. D.: GPS and ionosphere, in: Review of Radio Science (1996-1999), edited by: Ross Stone, W., Wiley-IEEE Press, New York, 625-665, 1999.

Mitchell, C. N. and Spencer, P. S. J.: A three-dimensional timedependent algorithm for ionospheric imaging using GPS, Annals of Geophysics, 46, 10 pp. doi:10.4401/ag-4373, 2003.

$\mathrm{Na}, \mathrm{H}$. and Lee, H.: Analysis of fundamental resolution limit of ionospheric tomography, in: Acoustics, Speech, and Signal Processing, 1992. ICASSP-92., 1992 IEEE International Conference on, 23-26 March 1992, San Francisco, CA, 97-100, 1992.

Natarajan, B. K.: Sparse Approximate Solutions to Linear Systems, SIAM J. Comput., 24, 227-234, doi:10.1137/S0097539792240406, 1995.

Schmidt, M.: Wavelet modelling in support of IRI, Adv. Space Res., 39, 932-940, doi:10.1016/j.asr.2006.09.030, 2007.

Schmidt, M., Bilitza, D., Shum, C., and Zeilhofer, C.: Regional 4-D modeling of the ionospheric electron density, Adv. Space Res., 42, 782-790, doi:10.1016/j.asr.2007.02.050, 2008.

Simons, F. J., Loris, I., Nolet, G., Daubechies, I. C., Voronin, S., Judd, J. S., Vetter, P. A., Charléty, J., and Vonesch, C.: Solving or resolving global tomographic models with spherical wavelets, and the scale and sparsity of seismic heterogeneity, Geophys. J. Int., 187, 969-988, doi:10.1111/j.1365-246X.2011.05190.x, 2011.
Spencer, P. S. J. and Mitchell, C. N.: Imaging of 3-D plasmaspheric electron density using GPS to LEO satellite differential phase observations, Radio Sci., 46, RS0D04, doi:10.1029/2010RS004565, 2011.

Sutton, E. and Na, H.: High resolution ionospheric tomography through orthogonal decomposition, in: Image Processing, 1994 Proceedings. ICIP-94, IEEE International Conference, 13-16 November 1994, Austin, TX, 148-152, 1994.

Tikhonov, A. N. and Arsenin, V. Y.: Solution of ill-posed problems, Scripta series in mathematics, Winston \& Sons, Washington, D.C., 258 pp., 1977.

Tsaig, Y. and Donoho, D. L.: Breakdown of equivalence between the minimal $\ell^{1}$-norm solution and the sparsest solution, Signal Process., 86, 533-548, doi:10.1016/j.sigpro.2005.05.028, 2006.

Yeh, K. C. and Raymund, T. D.: Limitations of ionospheric imaging by tomography, Radio Sci., 26, 1361-1380, doi:10.1029/91RS01873, 1991.

Zeilhofer, C., Schmidt, M., Bilitza, D., and Shum, C.: Regional 4-D modeling of the ionospheric electron density from satellite data and IRI, Adv. Space Res., 43, 1669-1675, doi:10.1016/j.asr.2008.09.033, 2009.

Zhdanov, M.: Geophysical Inverse Theory and Regularization Problems, in: Methods in Geochemistry and Geophysics, Elsevier, Eastbourne, 2002. 